\title{
A Cost-effective Liquid Core Waveguide based on a Concentrated Acid Medium for Colorimetric Determination of Sulfide
}

Wasin SOMBOOT, ${ }^{* 1, * 4}$ Jaroon JAKMUNEE, ${ }^{* 1, * 2, * 3}$ Tinakorn KANYANEE $* 1, * 2, * 3 \dagger$

${ }^{*}$ Department of Chemistry, Faculty of Science, Chiang Mai University, Chiang Mai, 50200, Thailand

${ }^{*}$ Center of Excellence in Materials Science and Technology, Chiang Mai University, Chiang Mai, 50200, Thailand

${ }^{*}$ Center of Excellence for Innovation in Chemistry, Chiang Mai University, Chiang Mai, 50200, Thailand

${ }^{*}$ The Graduate School, Chiang Mai University, Chiang Mai, Thailand, 50200, Thailand

$\dagger$ To whom correspondence should be addressed.

E-mail: tinakorn.kanyanee@cmu.ac.th,tkanyanee@gmail.com 


\begin{abstract}
A cost-effective long path absorption liquid core waveguide (LCW) device was fabricated from a typical Teflon tube and using a high refractive index (RI) medium of acid. It was incorporated in a flow injection-gas diffusion system for sensitive determination of sulfide via methylene blue (MB) chemistry. The gas diffusion unit could prevent some interferences in samples. The limit of detection of $0.13 \mu \mathrm{mol} \mathrm{L} \mathrm{L}^{-1}$ was achieved without using a pre-concentration procedure. The proposed device was successfully applied for sulfide determination in canal water samples with $88.7-103 \%$ recovery.
\end{abstract}

Keywords: Liquid core waveguide, Long path absorption spectrophotometry, Sulfide determination, Gas diffusion flow injection 


\section{Introduction}

A trace sulfide in an aquatic environment is an important parameter for environmental study. ${ }^{1}$ Various analytical methods for sulfide determination by using flow-based analysis were reviewed. ${ }^{1}$ For the absorption spectrophotometric method, methylene blue (MB) chemistry and nitroprusside (NP) chemistry have been commonly used. The method via MB chemistry was recommended as a standard method for determination of sulfide in water/wastewater. ${ }^{2}$ The MB chemistry in a flow injection system with laser/photodiodebased fluorescence detection was reported for sulfide determination, but the detector required an expensive laser module as an excitation light source. ${ }^{3}$ The in-line gas diffusion flow injection (GD-FI) system has been reported for the liberation/separation of the convertible gas analyte from the complicated sample matrices. ${ }^{4-5}$ Recently, the costeffective flow-batch setup with a porous membrane-based diffusion scrubber with the absorption spectrophotometry via NP chemistry was reported for sulfide determination in waters; however the lower detection limit was required for a trace level sulfide detection. ${ }^{6}$

A long path liquid core waveguide ( $\mathrm{LCW}$ ) cell has been used to improve the sensitivity of various spectroscopic techniques. ${ }^{7}$ The commercially available materials, Teflon-AF, was typically used in the LCW cells because the refractive index (RI) of Teflon AF (1.29) was lower than that of a typical aqueous core liquid (1.33); this leads to the occurring of total internal reflection (TIR) phenomenon inside the Teflon-AF capillary tube. ${ }^{8}$ The light is guided through the core liquid towards the end of the capillary, and as a result, the long-path optical measurement can be achieved. Although Teflon AF is convenient to fabricate for a long path absorption cell, it is expensive ( 420 \&USD/m). However, the alternative LCW approach can be performed without using Teflon-AF by modifying the core liquid medium with a solution that has higher RI than the typical tubing materials. Some organic solvents, such as carbon disulfide, as the high RI medium 
in the borosilicate tube as cladding material, was demonstrated as the modified LCW $(\mathrm{mLCW}){ }^{9-11}$ This approach offered the cost-effective mLCW cell for chemical analysis. Sucrose solution was suggested to promote the light-guiding phenomenon in a porous tube. ${ }^{12}$ Tsunoda et al. demonstrated the application of using $45 \% \mathrm{w} / \mathrm{w}$ ethanol for the light-guiding device and applied it for a long path absorption measurement of tryptophan. ${ }^{13}$ Teshima et al. applied a concentrated $\mathrm{NaOH}$ medium as an $\mathrm{mLCW}$ in the FEP tube for a long path absorption measurement for the acetone determination in exhaled breath. ${ }^{14}$ Recently, the syrup or ethanol was used as cost-effective environmentally friendly mLCW in a GD-FI system employing a butterfly pea flower extract reagent for ammonium determination. ${ }^{15}$

This work aims to adapt and utilize the high RI of the concentrated acid medium in $\mathrm{N}, \mathrm{N}$-dimethyl-p-phenylenediamine dihydrochloride (DMPD) reaction of the MB method to demonstrate as a simple trick for the cost-effective LCW for the sensitivity enhancement of sulfide determination without adding other reagents or pre-concentration procedures. The proposed acid medium-based LCW (aLCW) was adapted to use with an in-line GD-FI system to prevent the change of RI due to the matrices of the sample.

\section{Experimental}

\section{Reagents and Chemicals}

All chemicals are analytical reagent grade and were dissolved in deionized (DI) water. The desired concentration of $\mathrm{H}_{2} \mathrm{SO}_{4}$ was prepared by diluting the concentrated $\mathrm{H}_{2} \mathrm{SO}_{4}$ acid (98\%, Merck, Germany) with DI water. $2.0 \mathrm{~g} \mathrm{~L}^{-1}$ of DMPD solution was prepared by dissolving $0.50 \mathrm{~g}$ of the DMPD (Fluka, Switzerland) in $250 \mathrm{~mL}$ of $9 \mathrm{~mol} \mathrm{~L}^{-1} \mathrm{H}_{2} \mathrm{SO}_{4}$ as a working solution $\mathrm{A}$. The $16 \mathrm{mmol} \mathrm{L}^{-1}$ ammonium ferric sulfate solution, as an oxidizing reagent, was prepared by dissolving $1.95 \mathrm{~g}$ of $\mathrm{NH}_{4} \mathrm{Fe}\left(\mathrm{SO}_{4}\right)_{2} \cdot 12 \mathrm{H}_{2} \mathrm{O}$ (Qrec, New Zealand) 
in $250 \mathrm{~mL}$ of $0.1 \mathrm{~mol} \mathrm{~L}^{-1} \mathrm{H}_{2} \mathrm{SO}_{4}$ as a working solution B. Both solutions A and B were merged as an acceptor solution in the GD-FI, as depicted in Fig. 1. A standard stock solution of $25 \mathrm{mmol} \mathrm{L}^{-1}$ sulfide was prepared daily by dissolving $0.1952 \mathrm{~g}$ of sodium sulfide $\left(\mathrm{Na}_{2} \mathrm{~S} \cdot \mathrm{xH}_{2} \mathrm{O}\right.$, Loba chemie, India) in $50 \mathrm{~mL}$ of $25 \mathrm{mmol} \mathrm{L}-1 \mathrm{NaOH}$ to maintain the sulfide ion form. The exact concentration of sulfide was determined by using iodometric titration. The desired concentrations of the standard solutions were prepared by appropriately diluting the stock solution with $25 \mathrm{mmolL}^{-1} \mathrm{NaOH}$. The canal water samples were collected in the Chiang Mai area and preserved by adding $2.5 \mathrm{~mL}$ of $5 \mathrm{~mol}$ $\mathrm{L}^{-1} \mathrm{NaOH}$ for a $100 \mathrm{~mL}$ water sample to maintain the sulfide form and prevent $\mathrm{H}_{2} \mathrm{~S}$ gas forming, which may lose from the sample. The collected water samples were kept at $4^{\circ} \mathrm{C}$ in the refrigerator. ${ }^{2}$

\section{Apparatus and setup}

The 50-cm length aLCW cell was fabricated from $0.75 \mathrm{~mm}$ i.d. FEP tube (Upchurch Scientific, USA) integrated with two PEEK Tee-connectors, compatible with M6 nut/ ferrule for 1/16" o.d. FEP tube, $1.54 \mathrm{~mm}$ acrylic optical fiber, and $2 \mathrm{~mm}$ diameter sapphire ball (Edmund optics, Singapore), as demonstrated in Fig. 2 and Fig. S2 (supporting information). The red color bright LED (626 $\mathrm{nm} \lambda_{\max }$, Jamesco, USA) was used as a light source with $22.4 \mathrm{~mA}$ constant current drive. A low-cost photodiode with a built-in operational amplifier (TSL257, Mouser electronics, USA) was employed as a light sensor. The LED and photodiode were powered from a USB port or $5 \mathrm{~V}$ DC mobile phone charger. The aLCW cell was connected to the light source and photodiode through the acrylic optical fiber and fitted with the home-built acrylic connectors. The change of light intensity at the photodiode was monitored and converted to be an output voltage signal (0-5 V DC). The output signal was acquired to the computer through a low-cost 22-bit 
analog-digital converter data acquisition unit (Emant300, Emant, Singapore) with an inhouse software at $5 \mathrm{~Hz}$ sampling rate. The output signal was converted to the absorption unit via an equation; $\mathrm{Abs}=-\log \left(\mathrm{V}_{\mathrm{i}} / \mathrm{V}_{0}\right)$, where $\mathrm{Abs}=$ absorbance, $\mathrm{V}_{\mathrm{i}}=$ the recorded voltage signal, $\mathrm{V}_{0}=$ maximum voltage signal (baseline signal).

The aLCW cell was set to couple with a GD-FI system, as demonstrated in Fig. 1. The Teflon tube (0.75 i.d., $1.6 \mathrm{~mm}$ o.d., Upchurch Scientific, USA) was used throughout this work. The 3-channel peristaltic pump (Alitea, FIAlab Instrument, USA) was used as a propelling system. A 2-position-6-port injection valve with $442 \mu \mathrm{L}$ injection loop volume and a $20-\mathrm{cm}$ and $240-\mathrm{cm}$ length of mixing coils were used with the gas diffusion unit (GDU) with the dimension $\sim 30 \mathrm{~cm}$ membrane length, as explained in the previous work. ${ }^{14}$ A low-cost Teflon tape membrane, typically used in plumbing work, was used in the GDU.

\section{Results and Discussion}

The aLCW signal with various contents of acid solution

The transmittance signal of aLCW in the 50-cm path length FEP tube containing various concentrations of sulfuric or hydrochloric acid was evaluated in a constant temperature water bath as described in the previous work ${ }^{15}$, as depicted the results in Fig. 2. The concentration of sulfuric $>4 \mathrm{~mol} \mathrm{~L}^{-1}$ can provide a high $\mathrm{RI}$ enough to perform the TIR phenomenon. For the recommended flow-based MB method, ${ }^{2}$ the 9 mol L$^{-1} \mathrm{H}_{2} \mathrm{SO}_{4}$ was used as a medium of DMPD solution and merged with $\mathrm{Fe}^{3+}$ solution dissolved in 0.1

mol L $\mathrm{L}^{-1} \mathrm{H}_{2} \mathrm{SO}_{4}$; thus, the final concentration of the sulfuric acid solution was approximately $\sim 4.5 \mathrm{~mol} \mathrm{~L}^{-1}$. Therefore, this condition could provide the TIR phenomenon without adding other LCW modifiers. Again, the hydrochloric acid with the concentration 
$>4.0 \mathrm{~mol} \mathrm{~L}^{-1}$, which was used in the MB method, ${ }^{16-17}$ could be used in this approach as shown the transmittance signal in Fig. 2. It should be noted that the light-guiding intensity can be adjusted for an appropriate absorption range by changing the current to drive the LED or manipulate the flow rate of both $9 \mathrm{~mol} \mathrm{~L}^{-1} \mathrm{H}_{2} \mathrm{SO}_{4}$ and ferric solution to obtain the different final concentrations of the acid medium.

\section{The aLCW with GDU}

The preliminary test of the proposed aLCW system was carried out by using a double-line FIA system without using the GDU for total sulfide determination in water and wastewater, as recommended in the standard method, ${ }^{5}$ as demonstrated the manifold in Fig. S2 (supporting information). The 50-cm long-path absorption cell signal was set up and compared the signal with the 1-cm length Z-shape cell by serially arranging the two cells as described in Fig. S3 (supporting information). It was found that the sensitivity of aLCW cell increases approximately 50 times compared with using the Z-shape cell format. The LCW cell can reduce the Schlieren effect as described previously, ${ }^{18}$ The welldefined FI-gram can be obtained by using the long-path aLCW cell, and the linear relationship of the sulfide concentration and peak height signal was achieved in the range of $0.5-20 \mu \mathrm{mol} \mathrm{L} \mathrm{L}^{-1}$ sulfide ion. The limit of detection (LOD) and limit of quantitation (LOQ) based on three times and ten times of standard deviation of blank signal (SDblank) for this FI-manifold with the aLCW cell was 0.28 and $1.08 \mu \mathrm{mol} \mathrm{L}^{-1}$ sulfide (based on $66.3 \mu \mathrm{L}$ injection loop), respectively. The accuracy of the analytical system without using the GDU is present with \% recovery, as summarized in Table S1(supporting information). Without using the GDU, the sample was injected into the high concentration of the sulfuric acid of $9 \mathrm{~mol} \mathrm{~L}^{-1}$; this format would be helpful for the determination of total sulfide, including acid-volatile sulfide such as $\mathrm{H}_{2} \mathrm{~S}$, and acid-soluble metal sulfide, as 
described in the reference. ${ }^{2}$

By using the in-line GD-FI system combined with aLCW for sulfide determination, the method is based on the production of $\mathrm{H}_{2} \mathrm{~S}$ in an acidic medium of $0.5 \mathrm{M} \mathrm{H}_{2} \mathrm{SO}_{4}$ in the donor solution. The $\mathrm{H}_{2} \mathrm{~S}$ gas could diffuse through the porous membrane and dissolved into a mixture of DMPD and $\mathrm{Fe}^{3+}$ solution as an acceptor solution. The conversion of sulfide in donor solution to acceptor solution depends on i) conversion of sulfide to $\mathrm{H}_{2} \mathrm{~S}(\mathrm{aq})$ in the acid condition, which depends on the flow analysis conditions such as donor solution flow rate, mixing coil length, and acid concentration to lowering the $\mathrm{pH}$ level of the preserved solution $(\mathrm{NaOH})$, ii) the $\mathrm{H}_{2} \mathrm{~S}(\mathrm{aq}) / \mathrm{H}_{2} \mathrm{~S}(\mathrm{~g})$ equilibrium which depends on Henry's law, and iii) the dissolving of $\mathrm{H}_{2} \mathrm{~S}(\mathrm{~g})$ to form $\mathrm{S}^{2-}(\mathrm{aq})$ in the acceptor stream. ${ }^{5,6}$ This GD-FI system provides liberation of the $\mathrm{H}_{2} \mathrm{~S}$ gas analyte from the complicated matrices of the sample and prevents the change of the RI of the acceptor solution due to the sample matrices. The linear relationship of the sulfide concentration and peak height signal can be obtained in the range of $0.4-20 \mu \mathrm{mol} \mathrm{L}^{-1}$ sulfide ion with the equation of mAbs $=6.30 \pm 0.21\left[\mathrm{~S}^{2-}, \mu \mathrm{mol} \mathrm{L} \mathrm{L}^{-1}\right]+6.91 \pm 1.90, \mathrm{R}^{2}=0.9967$, as illustrated in FI-gram in Fig. 3. Without the use of the GDU, although the LCW can reduce the Schlieren effect, the blank signal appeared due to the change of refractive index (RI) of analyte/sample zone, while the LCW cell with the GDU system can eliminate the change of RI due to the samples solution. The lower blank signal can be obtained. The LOD and LOQ of the proposed aLCW cell with the in-line GDU system, based on three times and ten times of $\mathrm{SD}_{\text {blank, were }} 0.13$ and $0.44 \mu \mathrm{mol} \mathrm{L}^{-1}$ sulfide (based on $442 \mu \mathrm{L}$ injection loop), respectively. Although the analyte gas species cannot totally diffuse through the membrane, the use of the higher injection volume provides the lower LOD compared with the system without GDU. The repeatability of 7 replications was $3.3 \%$ RSD, as depicted in Fig. S5 (supporting information). The shorter FEP tube of $10-\mathrm{cm}$ 
length FEP tube was compared to the aLCW signal from the $50-\mathrm{cm}$ length FEP tube by serial arranging in the GD-FI system, as shown in the FI-grams of standard sulfide ion in Fig. S6 (supporting information). The linear relationship of the absorbance and sulfide concentration can be obtained in the range of $2-20 \mu \mathrm{mol} \mathrm{L} \mathrm{L}^{-1}$ sulfide for 10 -cm length aLCW with the linear equation of $\mathrm{mAbs}=1.29 \pm 0.06\left[\mathrm{~S}^{2-}, \mu \mathrm{mol} \mathrm{L} \mathrm{L}^{-1}\right]+1.21 \pm 0.76, \mathrm{R}^{2}=$ 0.9948. The 50-cm length aLCW cell provides higher sensitivity than that by using the 10 -cm length aLCW cell $\sim 5$ folds. In addition, the sensitivity of the analytical system could be improved by optimized the flow analysis conditions, such as injection loop length, donor solution flow rate, and appropriate mixing coil length, to provide the higher mass transport of sulfide $(\mathrm{g})$ across the membrane. ${ }^{16}$

\section{Application of the aLCW with the GD-FI for sulfide determination}

The in-line GD-FI system with the proposed aLCW cell was applied for sulfide determination in canal water samples containing low concentrations level of the analyte with the recovery of $88.7-103 \%$, as shown the result in Table 1 . The results indicated that the proposed method could be applied for trace sulfide determination in the canal water samples. The low limit of detection can be achieved with a long path absorption optical cell without using the pre-concentration unit. The proposed system would be applied for the determination of soluble sulfide since the low concentration of $\mathrm{H}_{2} \mathrm{SO}_{4}$ in the donor stream cannot dissolve metal sulfides, as explained in the previous work. ${ }^{5,6}$ Since the other convertible gas species in acid condition, such as $\mathrm{CO}_{3}{ }^{2-} / \mathrm{HCO}_{3}{ }^{-} / \mathrm{CO}_{2}(\mathrm{~g}), \mathrm{CN}^{-} / \mathrm{HCN}(\mathrm{g})$, and $\mathrm{SO}_{3}{ }^{2-} / \mathrm{SO}_{2}(\mathrm{~g})$ can diffuse through the hydrophobic PTFE membrane, the interfering species to the GD-FI system with absorption spectrometry via the MB method were studied and described in the previous article. ${ }^{5,16,17}$ There is no significant effect from other possible gas convertible species. Again, the EDTA was recommended as a masking agent 
for oxidizing species. ${ }^{5}$ The analytical performance of sulfide determination with GD-FI incorporated with the proposed aLCW was compared with other detection methods, as shown in Table S2. The sensitivity of the LCW could be further increased by using a smaller bore FEP tube for a longer path length or optimizing the flow analysis conditions such as sample injection loop or mixing coil length to provide the higher mass transport of sulfide across the membrane. ${ }^{16}$

\section{Conclusions}

It was successfully utilized the high $\mathrm{RI}$ of the of $\mathrm{H}_{2} \mathrm{SO}_{4}$ medium in the MB method to provide the TIR phenomenon in a cladding FEP tube to perform a cost-effective aLCW for a long path absorption spectrophotometry for a high sensitivity sulfide detection without adding other modifiers. The GDU serves to prevent the change of RI due to the sample matrices, and it was successfully applied for sulfide determination in canal water samples. Moreover, the proposed GD-FI with an mLCW system could be used for other chromogenic reagents with a higher RI medium, such as $\mathrm{CaCl}_{2}$ salt solution. ${ }^{14}$

\section{Acknowledgements}

This research project is supported by TSRI. The authors thank the Center of Excellence in Materials Science and Technology, Chiang Mai University, International Foundation for Science (IFS, W-5094-1), and Center of Excellence for Innovation in Chemistry (PERCH-CIC) for the partial financial support.

\section{Supporting Information}


Supporting information includes an absorption spectrum of methylene blue chemistry in $\mathrm{H}_{2} \mathrm{SO}_{4}$ medium (Fig. S1), photograph of the optical cell (Fig. S2), FI-manifold (Fig. S3), and the FI-gram (Fig. S4) of sulfide ion determination via MB chemistry without using GDU, \% recovery of the sulfide detection in water sample without using the GDU system, the repeatability of the GD-FI-aLCW via MB chemistry (Fig. S5), and the FI-gram with different lengths of cell (Fig. S6), comparison of the analytical performance of the proposed aLCW for sulfide determination with other systems (Table S2). This material is available free of charge on the Web at http://www.jsac.or.jp/analsci/.

\section{References}

1. L. Ferrer, M. Miro, J. M. Estela, V. Cerda, Trac-Trend. Anal. Chem., 2007, 26, 413.

2. Standard methods for the examination of water and wastewater, $22^{\text {nd }}$ revised ed., American Public Health Association, Washington DC, 2012. pp 4, 175-176.

3. M. A. Spaziani, J. L. Davis, M. Tinani, M. K. Carroll, Analyst, 1997, 122, 1555.

4. W. Somboot, J. Jakmunee, T. Kanyanee, Talanta, 2017, 170, 298.

5. V. Kuban, P.K. Dasgupta, J.N. Marx, Anal. Chem., 1992, 64, 36.

6. T. Khongrangdee, W. Somboot, J. Jakmunee, T. Kanyanee, Anal. Sci., 2020, 11, 1353.

7. L.J. Gimbert, P. J. Worsfold, Trac-Trend Anal. Chem., 2007, 26, 914.

8. R. N.M.J. Páscoa, I. V. Tóth, A. O.S.S. Rangel, Anal. Chim. Acta., 2012, 739, 1.

9. K. Fuwa, W. Lei, and K. Fujiwara, Anal. Chem., 1984, 56, 1840.

10. K. Fujiwara, K. Fuwa, Anal. Chem., 1985, 57, 1012.

11. K. Fujiwara, Anal. Chim. Acta.,1988, 212, 245.

12. P.K. Dasgupta, K. Petersen, Spectroscopy, 1987, 2(5), 50. 
13. K. Tsunoda, A. Nomura, J. Yamada, S. Nishi, Appl. Spectrosc., 1990, 44, 163.

14. N. Teshima, J. Li, K. Toda, P. K. Dasgupta, Anal. Chim. Acta., 2005, 535, 189.

15. W. Somboot, J. Jakmunee, T. Kanyanee, Microchem. J., 2020, 159, 105555.

16. G. de Armas, L. Ferrer, M. Miró, J.M. Estela, V. Cerdà, Anal. Chim. Acta., 2004, 524, 89.

17. L. Ferrer, J.M. Estela, V. Cerdà, Anal. Chim. Acta., 2006, 573-574, 391.

18. P. S. Ellis, B. S. Gentle, M. R. Grace, I. D. McKelvie, Talanta, 2009, 79, 830. 
Table 1 The recovery of sulfide determination in canal water samples by using the GDFI coupled with aLCW detection.

\begin{tabular}{|c|c|c|c|}
\hline Samples & $\begin{array}{l}\text { Added } \\
/ \mu \mathrm{mol} \mathrm{L}-1\end{array}$ & $\begin{array}{l}\text { Found }^{\mathrm{a}} \\
/ \mu \mathrm{mol} \mathrm{L}{ }^{-1}\end{array}$ & $\begin{array}{c}\text { Recovery, } \\
\%\end{array}$ \\
\hline \multirow[t]{3}{*}{ Canal water \#1 } & 0 & $2.4 \pm 0.4$ & - \\
\hline & 10 & $11.6 \pm 1.1$ & 92 \\
\hline & 20 & $22.1 \pm 0.5$ & 98 \\
\hline \multirow[t]{3}{*}{ Canal water \#2 } & 0 & $1.8 \pm 0.1$ & - \\
\hline & 10 & $10.8 \pm 1.2$ & 90 \\
\hline & 20 & $21.4 \pm 0.9$ & 98 \\
\hline \multirow[t]{3}{*}{ Canal water \#3 } & 0 & $2.3 \pm 0.4$ & - \\
\hline & 10 & $12.0 \pm 0.6$ & 97 \\
\hline & 20 & $22.6 \pm 1.3$ & 101 \\
\hline \multirow[t]{3}{*}{ Canal water \#4 } & 0 & $2.0 \pm 0.1$ & - \\
\hline & 10 & $10.9 \pm 0.9$ & 89 \\
\hline & 20 & $22.6 \pm 1.3$ & 103 \\
\hline
\end{tabular}

${ }^{a}$ Average \pm standard deviation of triplicated results. 


\section{Figure Captions}

Fig. 1 Schematic diagram of the GD-FI system with aLCW for sulfide ion determination via $\mathrm{MB}$ chemistry.

Fig. 2 a) LED-based aLCW absorbance flow cell, b) the transmittance signal of the liquid core waveguide through high $\mathrm{RI}$ medium of $\mathrm{H}_{2} \mathrm{SO}_{4}$ and $\mathrm{HCl}$ at $30^{\circ} \mathrm{C}$ by using a red-LED with $2 \mathrm{~mA}$ current drive.

Fig. 3 The GD-FI-gram of 50-cm path length of absorption aLCW and a linear calibration graph of sulfide ion determination via MB method; $442 \mu \mathrm{L}$ sample injection volume, Acceptor solution: DMPD $2.0 \mathrm{~g} \mathrm{~L}^{-1}$ in $9 \mathrm{~mol} \mathrm{~L}^{-1} \mathrm{H}_{2} \mathrm{SO}_{4}$, with the flow rate of $0.25 \mathrm{~mL}$ $\min ^{-1}$, and $16 \mathrm{mmol} \mathrm{L}^{-1} \mathrm{Fe}^{3+}$ with the flow rate of $0.25 \mathrm{~mL} \mathrm{~min}^{-1}$, mixing coil 20 as 240 cm, $0.5 \mathrm{~mol} \mathrm{~L}^{-1} \mathrm{H}_{2} \mathrm{SO}_{4}$ as donor solution with the flow rate of $0.5 \mathrm{~mL} \mathrm{~min}^{-1}$ with a mixing coil length of $50 \mathrm{~cm}$. 


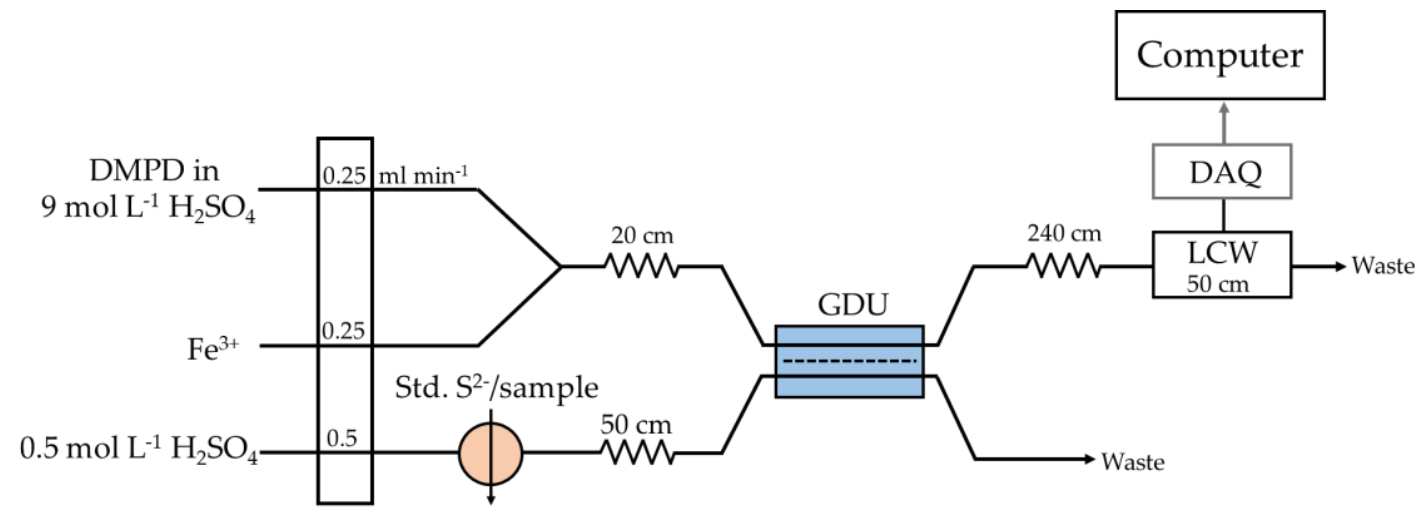

Fig. 1 Schematic diagram of the GD-FI system with aLCW for sulfide ion determination via MB chemistry. 
a
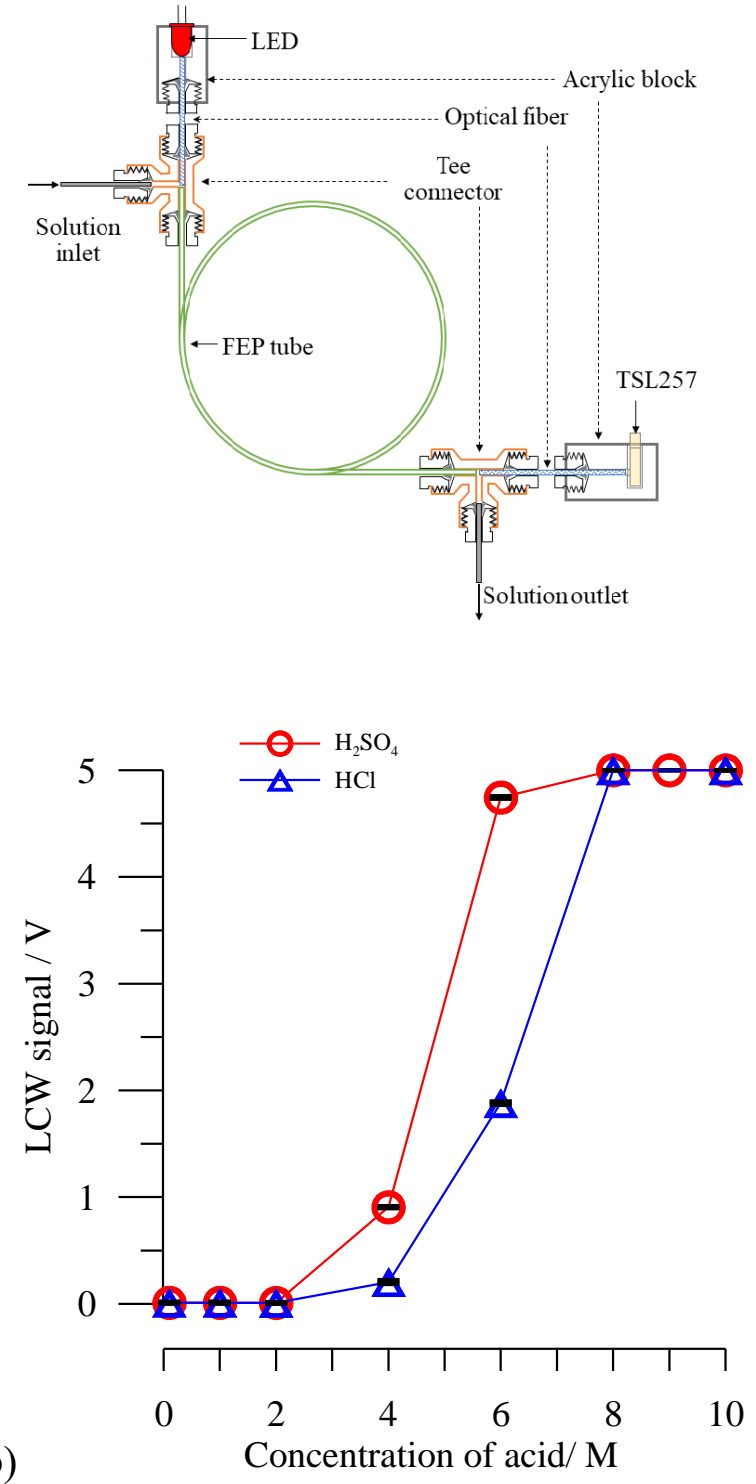

Fig. 2 a) LED-based aLCW absorbance flow cell, b) the transmittance signal of the liquid core waveguide through high $\mathrm{RI}$ of $\mathrm{H}_{2} \mathrm{SO}_{4}$ and $\mathrm{HCl}$ at $30^{\circ} \mathrm{C}$ by using a redLED with $2 \mathrm{~mA}$ current drive. 


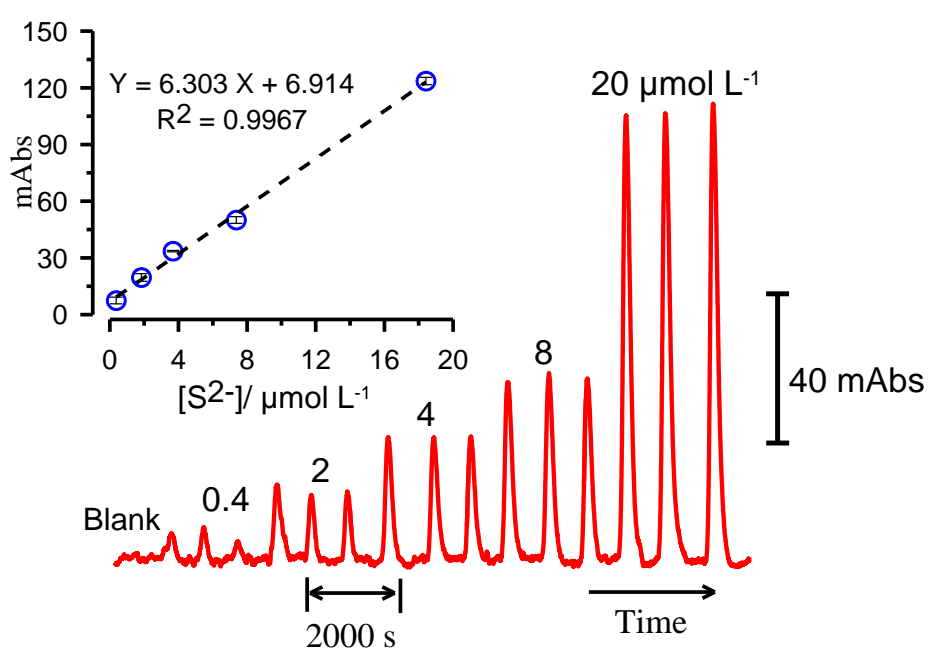

Fig. 3 The GD-FI-gram of 50-cm path length of absorption aLCW and a linear calibration graph of sulfide ion determination via MB method; $442 \mu \mathrm{L}$ sample injection volume, Acceptor solution: DMPD $2.0 \mathrm{~g} \mathrm{~L}^{-1}$ in $9 \mathrm{~mol} \mathrm{~L}^{-1} \mathrm{H}_{2} \mathrm{SO}_{4}$, with the flow rate of $0.25 \mathrm{~mL}$ $\mathrm{min}^{-1}$, and $16 \mathrm{mmol} \mathrm{L}^{-1} \mathrm{Fe}^{3+}$ with the flow rate of $0.25 \mathrm{~mL} \mathrm{~min}^{-1}$, mixing coils of 20 and $240 \mathrm{~cm}, 0.5 \mathrm{~mol} \mathrm{~L}^{-1} \mathrm{H}_{2} \mathrm{SO}_{4}$ as a donor solution with the flow rate of $0.5 \mathrm{~mL} \mathrm{~min}^{-1}$ with a mixing coil of $50 \mathrm{~cm}$ length. 


\section{Graphical Index}

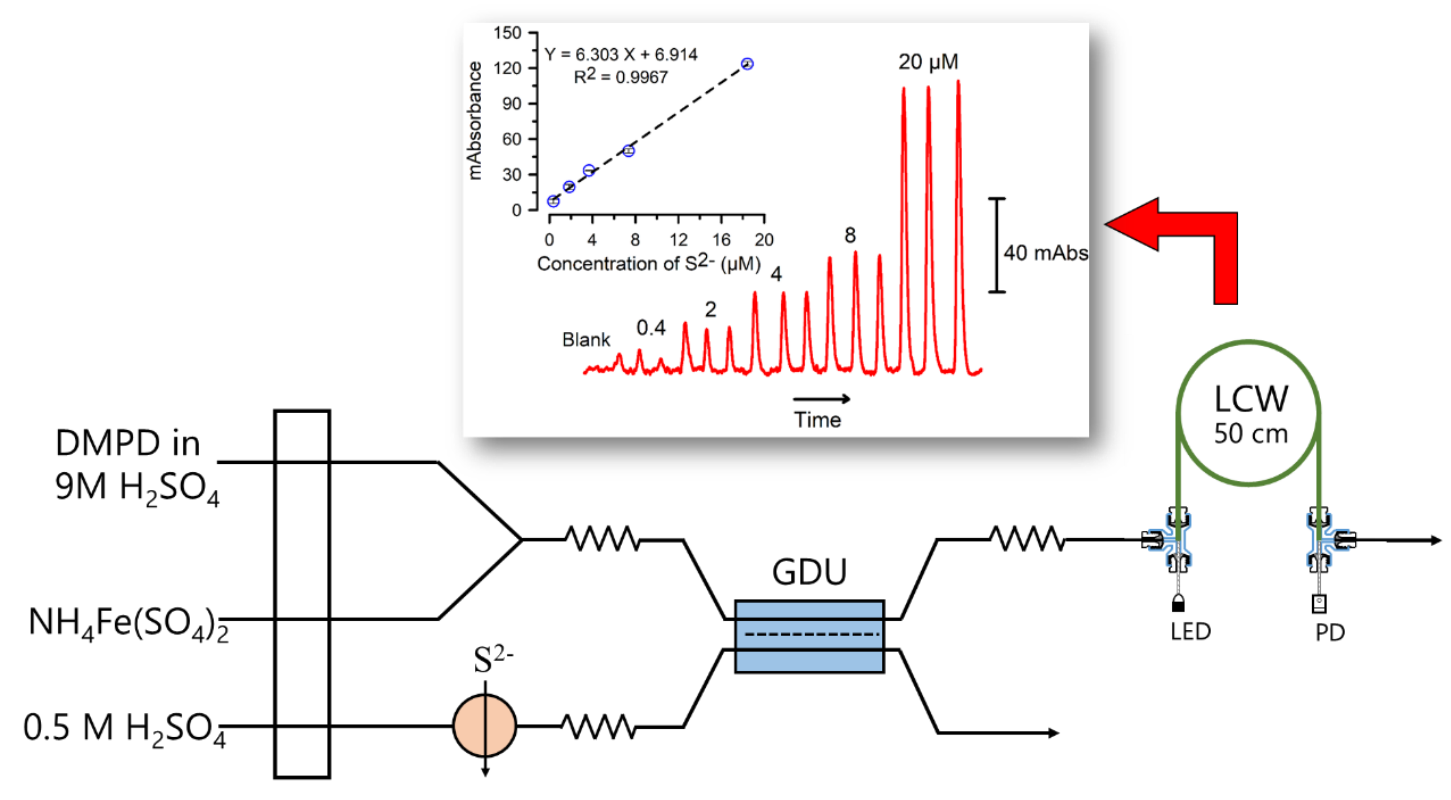

